\title{
Dominance, the bidirectional hypothesis, and Pavlovian backward conditioning in the US-US paradigm
}

\author{
DAVID S. TUBER \\ The Ohio State University, Columbus, Ohio
}

\begin{abstract}
Based upon considerations raised by Soviet research, the role of relative stimulus intensity, or dominance, in the unconditioned stimulus-unconditioned stimulus (US-US) paradigm was investigated under circumstances presumed favorable to the backward conditioned response (CR). Using the classically conditioned forelimb response of the cat, a brief shock (US1) delivered to one forepaw preceded a shock (US2) to the opposite forepaw in paired conditioning fashion; subjects in the control group received explicitly unpaired presentations of the stimuli. Conditioning in both the forward and backward directions was evaluated by the appearance of contralateral CRs on test trials to each of the USs. In Experiment 1, a ratio of the intensities between US1 and US2 of 100:80 was used to create a relative dominance in favor of the backward CR. In addition, to evaluate the suggestion that the appearance of the backward CR is retarded in the Pavlovian paradigm, overtraining was provided to a forward conditioning criterion of $200 \%$. In Experiment 2, the cats were exposed to successive reductions in the intensity of US2 to verify manipulations of dominance reportedly involved in the reactivation of a latent backward CR. Although forward conditioning was readily established to US1, there was no evidence of backward conditioning to US2 under any of the conditions.
\end{abstract}

Despite the preeminence of the traditional Pavlovian paradigm as a model for the associative process, its failure to provide evidence for the backward conditioned response (CR) stands in marked contrast to a growing body of support based upon methodologies in which the effects of unconditioned stimulus-conditioned stimulus (US-CS) pairings are assessed by their modification of an instrumental behavior (cf. Spetch, Wilkie, \& Pinel, 1981). With the exception of Wagner and Terry's (1975) results, the results of Pavlovian backward conditioning in this country have been negative; have revealed inhibitory processes; or, in apparently successful experiments, have failed to withstand the challenge of an evolving control methodology (see reviews by Cautela, 1965; Hall, 1976, 1984; Mackintosh, 1974). Whether this discrepancy reflects merely a difference in sensitivity between the two paradigms or a distinguishing feature of different processes is not clear. In this area of research, few broad parametric investigations have been undertaken (Switzer, 1930; Wolfle, 1932); few have been guided by theoretical considerations (Champion \& Jones, 1961; Trapold, Homzie, \& Rutledge, 1964; Wagner \& Terry, 1975).

\footnotetext{
This report is dedicated to the late Carol Dow Wickens.

This research, undertaken in partial fulfillment of the requirements for the PhD degree (1979) at The Ohio State University, was supported by NIMH Grant No. MH-08423 awarded to Delos D. Wickens. I am greatly indebted to Professor Wickens, academically and otherwise. Requests for reprints should be sent to the author, Department of Psychology, The Ohio State University, 142 Townshend Hall, 1885 Neil Avenue Mall, Columbus, $\mathrm{OH}$ 43210-1222.
}

Underscoring these limitations is the fact that although Soviet efforts with the conventional US-CS paradigm have fared little better than those in the West, Soviet researchers' variations on the procedure have apparently been more fruitful (Beritoff, 1965; Razran, 1956). Although these variations have rarely been tested against a control procedure, the circumstances under which Soviet conditioning has been successful (characterized by a US that is not so strong that it completely dominates the CS) suggest that the ratio of the intensity between the stimuli being paired is a critical parameter for effective conditioning (Razran, 1956, 1957). Moreover, the extant Soviet statement on the backward CR, the theoretical formulation of conditioning known as the bidirectional hypothesis (Asratyan, 1965, 1967; Beritoff, 1965), which elaborates on the conditions favorable to the backward CR, provides both a methodology and a conceptual schema for reexamining the phenomena.

The notion that associative connections are bidirectional, proceeding not only from the CS to the US but from the US to the CS, is predicated upon the demonstration of conditioning in a unique paradigm in which each of the stimuli being paired elicits an observable response. Thus, when a forelimb shock is paired with food, the shock comes to elicit the salivary CR in typical forward fashion; the presentation of food elicits the leg lift in the backward direction. Although, relative to the forward CR, the backward response is acknowledged to be fragile, its appearance and strength are reportedly maximized under conditions in which the stimuli being paired are biologically significant and are arranged so that the 
intensity of the first stimulus exceeds that of the second (Asratyan, 1965).

Conceptually, the absolute intensity of the stimuli being paired is presumed to influence the overall level of cortical excitability by determining the number of connections available between the cortical centers for each stimulus. Thus CRs in both directions are observed to be weakest with CS-CS pairings, and strongest with US-US pairings. The strength of the individual connections, and hence the directionality of conditioning, is presumably determined by the relative dominance of one cortical center over the other. Thus, variations in stimulus intensity that potentiate the relative strength of the first stimulus are observed to produce a corresponding enhancement of the backward CR-a result viewed as reflecting a modification in the balance of the relative excitability between the cortical centers (Asratyan, 1965).

In the single test of the bidirectional hypothesis in this country (see, however, Stephens, 1934; Wendt, 1930), Gormezano and Tait (1976) paired oral injections of water (USw) and airpuffs to the eye (USa) in the conditioning of the rabbit's jaw movement and nictitating membrane response. Comparisons between groups that received USw-USa pairings and groups that received USa-USw pairings revealed equivalent levels of forward conditioning but differential performance in the backward CR, depending on the order of the stimuli being paired. With water as the first stimulus (USw-USa), the jaw movement CR elicited by the airpuff exhibited a typical acquisition function; under the reverse order of pairing (USa-USw), the nictitating membrane response elicited by the water US showed an extinction-like function similar to that described by Spooner and Kellogg (1947). Although, theoretically, such differences could be accounted for on the basis of a more intense water US, the appearance of an uncharacteristically high level of responding among the explicitly unpaired controls precluded an associative interpretation. Nevertheless, the orderliness of the functions among the experimental groups, statistically compromised perhaps by the vulnerability of the jaw movement response to pseudoconditioning (Sheafor, 1975), raises the possibility that nonassociative factors arising within the controls are potentially obscuring the presumably fragile backward CR. This suggests that one of the features of Wagner and Terry's (1975) study, the use of experimentally sophisticated subjects, may have contributed significantly to their success by providing the sensitivity necessary for the detection of conditioning.

\section{EXPERIMENT 1}

In view of the limited research on the US-US paradigm and the potential importance of a theoretical schema appropriate to both forward and backward conditioning, the present research was designed to address the considerations raised by Asratyan $(1965,1967)$ and Razran (1956). Together, these views suggest that the conditions favorable to the appearance of a backward CR are the pairing of two relatively strong stimuli, presented so that the intensity of the first exceeds that of the second by some optimal value.

A stringent test of the hypothesis and the role of dominance requires the a priori specification of the intensive properties of the stimuli being paired. The use of brief shocks, as they were employed for hand-withdrawal conditioning in humans (Stephens, 1934), provides this advantage; in the present study, involving the cat's forelimb flexion response, a shock to one forepaw (US1) preceded a shock to the other forepaw (US2) in paired conditioning fashion. Thus the appearance of a contralateral CR with each US on test trials affords a direct assessment of the bidirectionality of conditioning. ${ }^{1}$ In addition to avoiding the difficulty of equating the impact of stimuli from different modalities, the use of otherwise identical shocks permits a comparison of the development of the forward and backward CRs within the same subject. To maximize the appearance of the backward CR, the intensity of US1 was arranged to exceed that of US2. In the absence of formal data on appropriate ratios for such conditioning, a US1:US2 ratio of $100: 80$ was selected to provide a nominal dominance in favor of the backward connection.

An ancillary issue addressed in the present experiment concerned the amount of training required for the development of the backward CR. Pavlov's (1927, p. 393) revised view that the backward $C R$ is transitory and limited to the early stages of training finds support in current research with conditioned suppression (Heth, 1976; Mahoney \& Ayres, 1976). In contrast, however, both Beritoff (1965) and Asratyan (1972) have reported that the backward CR may benefit from extended training - a view that finds some support in research with the Pavlovian procedure (Harlow, 1939; Wagner \& Terry, 1975). Consequently, protracted training was provided in the present experiment to evaluate the possibility that in the classical paradigm, the development of the backward $C R$ is retarded.

\section{Method}

Subjects. The subjects were 12 adult cats: 8 intact males and 4 spayed females. All cats had previously served as subjects in classical conditioning experiments involving the conditioned forelimb response established to a neutral stimulus (D. D. Wickens, Tuber, Nield, \& C. D. Wickens, 1977).

Apparatus. The method of restraint for the cats has been described in detail by D. D. Wickens, Meyer, and Sullivan (1961). Briefly, the cat is supported in a nylon mesh sling suspended from a rod $38 \mathrm{~cm}$ above the surface of a table. The cat's legs extend through four holes in the sling and are moderately restrained to fixed points on the table. This provides a degree of support for the cat and ensures that although the legs are free to move, they can be reasonably equated for degree of extension. Strain gauges were attached to the restraints on the forelimbs to detect movements which were then amplified by Hewlett-Packard preamplifiers and recorded on a Sanborn 320 recorder. Two Applegate 250 stimulators provided the shock stimuli, which were applied to the forepaws through electrodes. The electrode sites were shaved weekly; prior to each session, they were cleaned with alcohol and prepared with an electro- 
lytic cream. Timing of the stimulus events was controlled by Tektronix pulse and waveform generators. The cat was exposed to the experimental treatment in a Model $\mathbf{4 0 2}$ double-walled Industrial Acoustics chamber. The chamber was dimly illuminated and $50 \mathrm{~dB}$ SPL white noise provided a stable background and masking sound. An observer remained inconspicuously in the chamber with the cat to monitor the experiment and to ensure equality of the tension on the strain gauges.

US1 and US2 were brief shocks of $60 \mathrm{msec}$ duration. US1 was programmed to be delivered to one forepaw, US2 to the other. The intensity of US1 for each cat was the intensity determined from the cat's prior conditioning experience to be sufficient to support forward conditioning to a neutral stimulus. Descriptively, it was the minimum amount of shock necessary to produce a brisk, nonhabituable flexion of the forelimb without disturbing the complacency of the cat. The intensity of US2 for each cat was set at $80 \%$ of the value determined for US1; thus, the US1:US2 ratio was 100:80. The actual US1 values used in this study averaged $2.5 \mathrm{~mA}$ and ranged from 1.6 to $4.1 \mathrm{~mA}$. For the cats eventually designated as the experimental group, the average was $2.46 \mathrm{~mA}$ (range 1.6-4.1 mA); for the cats assigned to the control condition, the average was $2.5 \mathrm{~mA}$ (range 2.1-3.5 mA).

Procedure. Based on their prior experimental history, the cats were distributed into three squads of 4 cats. Within each squad, pairs of cats were matched on the basis of their previously demonstrated rate of learning and one member of each pair was then randomly assigned to each condition (experimental and matched control). In order to minimize any systematic effect of prior response learning on either the forward or the backward CR, the pairs were counterbalanced with respect to the paw designated to receive US1.

The cats in the experimental group received daily conditioning sessions consisting of 12 trials each. For 10 of the trials, US1 and US2 were presented in paired fashion at an interstimulus interval (ISI) of $750 \mathrm{msec}$. The 2 remaining trials were designated as tests for forward and backward conditioning and consisted of one interspersed presentation of US1 alone (the test for the forward CR) and one presentation of US2 (the test for the backward CR). The ordinal position of these test trials varied across the 5-day experimental week, with the order of precedence of the US1 and US2 test trials alternating between sessions. The intertrial interval averaged $45 \mathrm{sec}$ and ranged from 30 to $60 \mathrm{sec}$ in steps of $15 \mathrm{sec}$. Each cat remained on this conditioning regimen for a period of training equal to three times the number of trials required for the cat to attain a forward conditioning criterion of four CRs out of six consecutive test trials to US1.

Each cat assigned to the control group received the same number of US1 and US2 presentations each session, and the same number of sessions, as did the cat in the experimental group with which it was matched. The stimuli, however, were presented in explicitly unpaired fashion on a quasi-random schedule of occurrence such that no stimulus could occur more than twice in succession. Five basic schedules were devised that provided for the appearance of a US1 or US2 event at average intervals of $25 \mathrm{sec}$ (intervals ranged from 10 to $50 \mathrm{sec}$ in $5-\mathrm{sec}$ steps). The positions of the designated test trials for US1 and US2 corresponded to the ordinal positions of the test trials being sampled in the experimental group.

Immediately prior to the start of the experiment, the cats were reintroduced to the experimental chamber for three sessions, one per day, during which they were simply placed briefly into the restraining sling, suspended, and refamiliarized with the electrodes and strain gauges. On Day 4, each cat was exposed to four presentations of US1, followed on Day 5 by four presentations of US2. Each stimulus was delivered to the appropriate paw and at the intensity designated for that paw. The conditioning and control procedures were then initiated on the first day of the subsequent week and were continued until each subject achieved the overtraining criterion.
Conditioned forelimb responses, which typically appear as large and distinctive excursions of the polygraph pen, were judged independently by two experienced raters (D. D. Wickens, Nield, Tuber, \& C. D. Wickens, 1969). Uncertainty with respect to the latency and form characteristics of the backward CR, however, led to the application of a liberal criterion; the raters accepted those movements occurring between 200 and 1,500 msec after the presentation of the test stimulus that were judged to have deviated in form and magnitude from the prevailing pattern of background activity.

\section{Results and Discussion}

Figure 1 depicts the mean percent responding on the test trials for forward and backward conditioning in both the experimental and control groups. Since the cats in the experimental group attained the terminal criterion at different rates, their performance and that of their matched controls have been distributed into successive sixths of training for comparison.

As can be seen in Figure 1, forward conditioning as measured on the US1 test trials was the most prominent feature of the experiment. The average number of sessions required to attain the forward conditioning criterion of $67 \%$ responding was 12.8 sessions (128 trials; range, 5 to 32 sessions). Correspondingly, the total length of training for individual cats and their respective controls ranged from 15 to 96 sessions.

Application of the Mann-Whitney $U$ test confirmed that the performance of the experimental group departed significantly from that of the control group at the third sixth

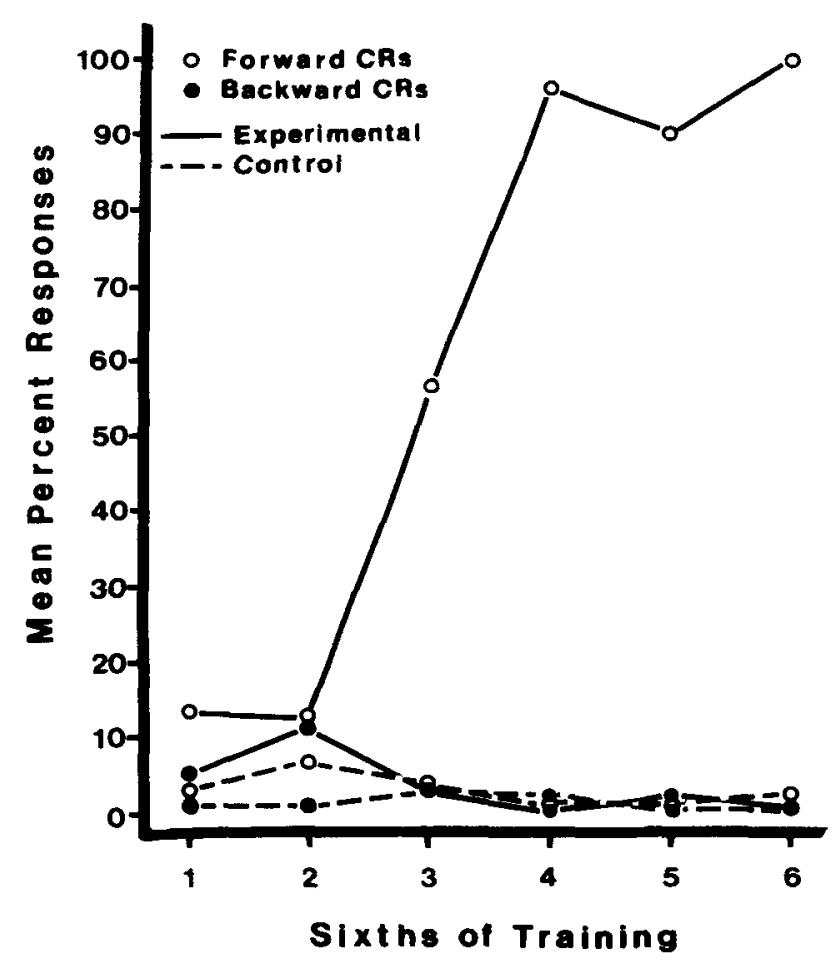

Figure 1. Acquisition of conditioned responses as measured on test trials to US1 (forward conditioning) and to US2 (backward conditioning) for experimental and explicitly unpaired control groups. 
of training and remained so at subsequent stages of training $(\mathrm{U}=1, p=.002)$.

The mean latency for the forward CR exhibited only minor fluctuations over the course of conditioning. The mean latency obtained during the final sixth of training (620 msec; range 540 to $660 \mathrm{msec}$ ) is consistent with conventional leg flexion conditioning using a neutral CS (Wickens et al., 1969).

Similar comparisons of the mean performance of the experimental and control groups on the tests for backward conditioning were uniformly nonsignificant. Although the small number of subjects responding precludes a detailed analysis of the data, the directionality of the differences observed within the matched pairs on parameters independent of the averaging bias introduced by vincentization (number of trials to first CR and total number of responses) equally favored the experimental and control conditions.

These results are consistent in demonstrating yet another failure to obtain backward conditioning with a traditional Pavlovian paradigm. Despite the fact that dominance, as defined by US intensity, favored the development of the backward connection, it is clear that the influence of temporal priority prevailed.

It is also apparent that the absence of backward conditioning cannot reasonably be ascribed to a failure to provide sufficient training. Under the conditions of this experiment, the extended conditioning criterion of $200 \%$ overtraining seems to allow ample opportunity to observe any retarded development of the backward CR. Nor can the failure be attributed to a confounding by the level of performance in the control groups: The paw withdrawal response, due either to its inherently low sensitivity to pseudoconditioning or to the experimental sophistication of the cats, provided a sufficiently unobtrusive background against which to assess any excitatory processes in the paired, experimental condition. An example of the cat's forepaw behavior during conditioning and testing is presented in Figure 2.

\section{EXPERIMENT 2}

One of the most compelling arguments presented by Asratyan (1965) in support of the role of the differential excitability of the cortical centers in the initiation and maintenance of the backward CR involves the manipulations of US intensities that either potentiate an existing backward CR or reactivate one that is latent. In a study by Liang Ji-An (cited in Asratyan, 1965), a shock to a dog's foreleg was paired with food at an ISI of $10 \mathrm{sec}$. The backward CR, a leg lift elicited by the presentation of food, proved to be the weaker connection and, with continued training, eventually disappeared. Reactivation of the CR, however, was possible by either of two methods: increasing the intensity of the US1 shock, or reducing the impact of the food US2 by satiating the dog prior to the experiment. In both cases, according to Asratyan, the resulting effect was to change the relative dominance of the center for US1, thereby reinstating the backward CR. It is the latter manipulation that is particu-
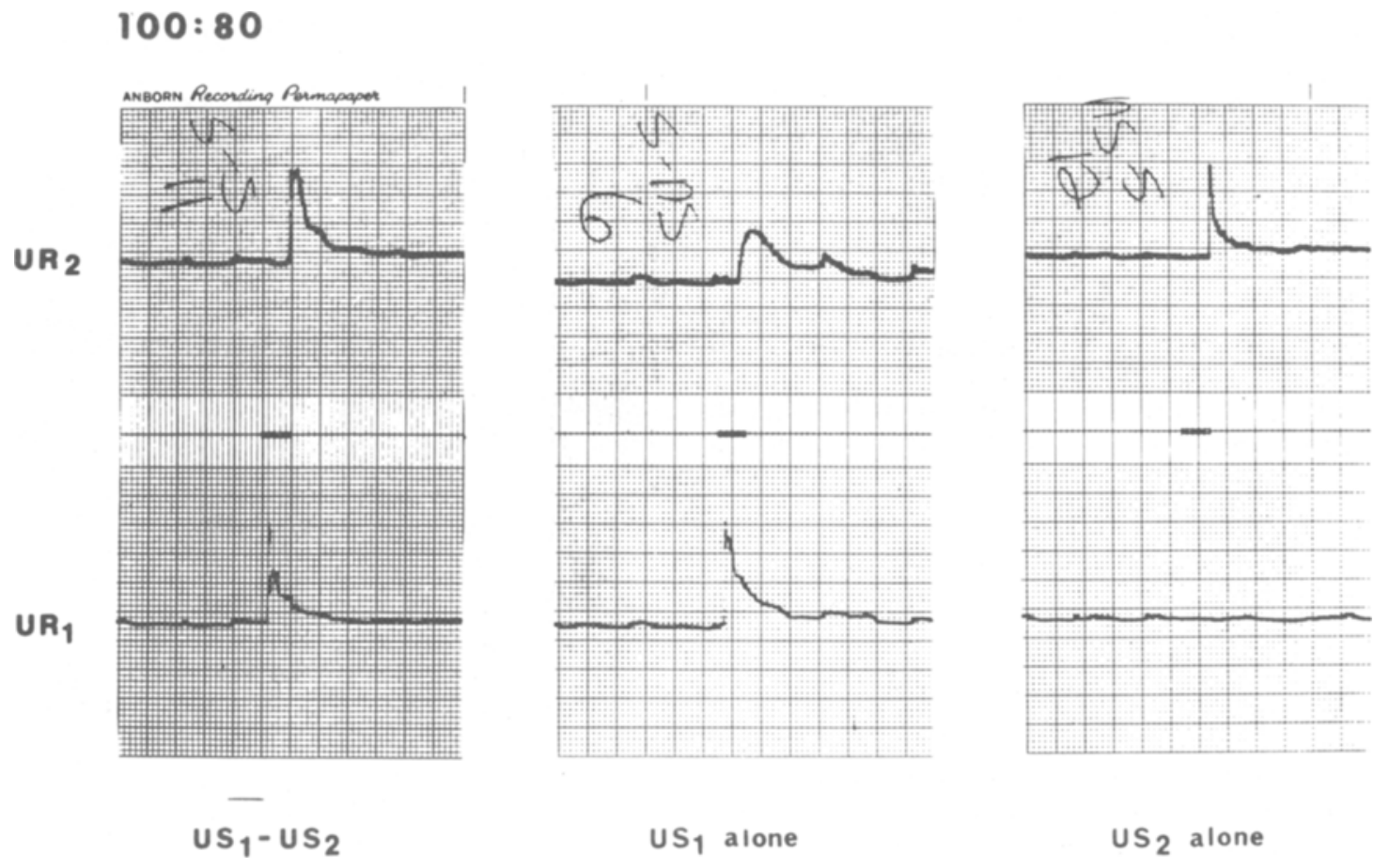

Figure 2. Characteristic appearance of unconditioned responses (URs) during conditioning (left panel); on test trials in response to US1 (middle panel); and in response to US2 (right panel). For the cat whose responses are shown, US1 was delivered to the left forepaw, US2 to the right. 
larly attractive, because with the reduction of the intensity of US2, and hence a reduction in the overall intensity of the paired compound, any emerging CR is immune to arguments that appeal to the process of sensitization in promoting the reinstatement.

Moreover, the failure to obtain conditioning in Experiment 1 may be attributable to the selection of an inappropriate US1:US2 ratio. If the ratio was inappropriate, the relative excitability of US1 may not have been sufficiently different from that of US2 to initiate the backward $\mathrm{CR}$, and the forward CR may have prevailed from the onset of conditioning. In view of the results of Liang JiAn's experiment, exposing the cats of Experiment 1 to reductions in the intensity of US2 should remedy any potential imbalance between US1 and US2 and provide a test of the lability of relative dominance.

\section{Method}

Subjects. The subjects were five of the six pairs of cats used in Experiment 1. The sixth pair was excused from the experiment due to differential experience following their participation in Experiment 1. periment 1 .

Procedure. Immediately following the completion of the terminal criterion for Experiment 1, the cats were exposed to successive reductions in the intensity of US2 on the following schedule: four sessions with a US1:US2 ratio of 100:60; four sessions with a ratio of 100:40; and four sessions with a ratio of 100:20. Exposure to the latter ratio, and the experiment proper, was concluded when the performance of the forward CR decreased to a level of no more than one CR on four successive test trials for the forward CR. All other conditions of training and testing were the same as described for Experiment 1.

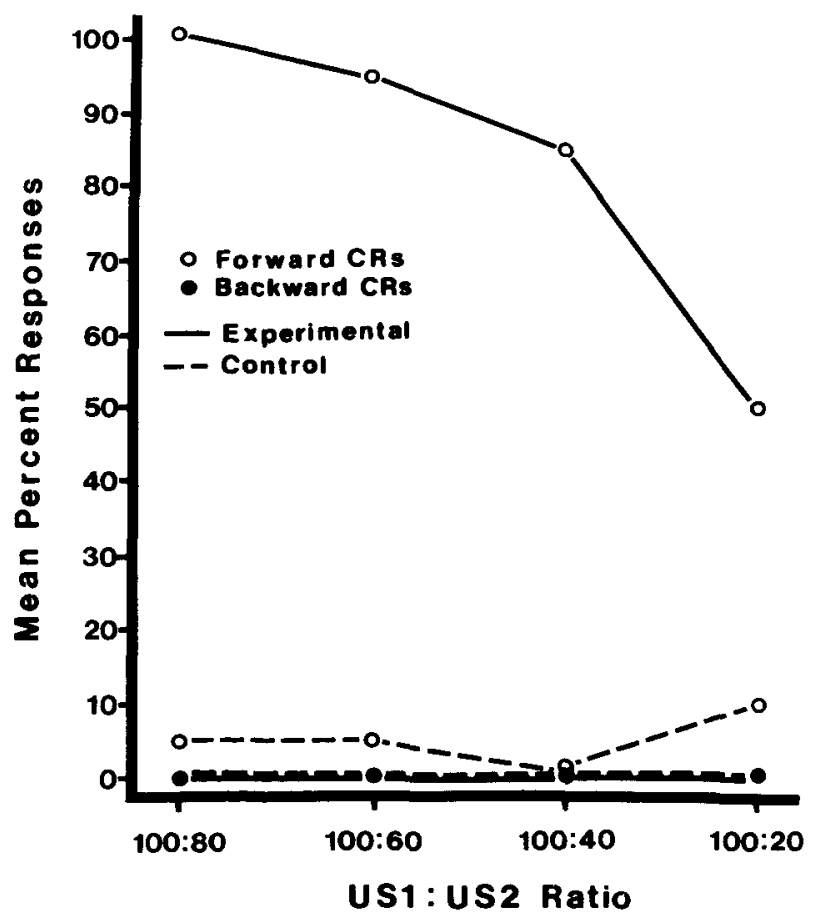

Figure 3. Effect of successive reductions in US2 intensity on the maintenance of the conditioned response as measured on test trials to US1 and US2.

\section{Results and Discussion}

Figure 3 reflects the effect of successive reductions in US2 intensity on conditioning at each of the three stages, with reference to the final 4 sessions of Experiment 1 at the 100:80 ratio. Due to the differential attrition of the cats as they reached the terminal extinction criterion, only the first 4 sessions of $100: 20$ are shown. Only 2 cats achieved the extinction criterion within the first 4 sessions at 100:20; the criterion was achieved by all cats in an average of 6.6 sessions (range 4-11 sessions). An analysis of variance performed on the forward conditioning tests across the four levels of US2 intensity was significant $[F(3,12)=17.7, p<.01]$. A post hoc analysis indicated that only at the 100:20 ratio was performance significantly different $(p<.01)$.

The results of Experiment 2 do not corroborate the findings of Liang Ji-An (cited in Asratyan, 1965). Reducing the intensity of US2 did not have the effect of restoring the backward connection-assuming, of course, that it was present in the first place. Instead, the consequence of such a reduction is a gradual weakening of the forward $C R$, an effect that is consistent with the general literature on US intensity and conditioning with a neutral CS.

\section{GENERAL DISCUSSION}

These experiments sought to directly accommodate the various conditions and conceptions implicated in the initiation and maintenance of the backward CR, principally those of Asratyan $(1965,1967)$ and Razran $(1956,1957)$. Among the most salient features of the present research was the ability to specify, a priori, the relative intensity of the stimuli being paired and to manipulate the degree of dominance in an attempt to maximize the appearance of the backward CR. In addition, testing for both forward and backward conditioning occurred throughout all stages of a very protracted training regimen ensuring that all views on the temporal origin of the CR could be satisfied and evaluated. Finally, the stability of the paw withdrawal response system provided the necessary sensitivity in the control condition against which any such associative effects could have been detected. Yet despite these considerations, the results of both experiments were markedly negative.

Two principal reasons have traditionally been offered for the failure of backward conditioning. The first, initially proposed by Pavlov $(1927$, p. 392), attributes the failure to the disruptive effects of external inhibition, or to a masking produced by the intensity of the first stimulus on the second. However, the US-US paradigm, as used in the present report, argues against this point: Reception of the second stimulus is reflected overtly as the unconditioned response, and its presence on each training trial ensures that the stimulus is at least available for the potential association. More importantly, the fact that forward conditioning occurred within the US-US procedure offers direct verification that US2 was indeed having a central effect. 
The second reason regards the selection of inappropriate US1:US2 ratios (Razran, 1956). The successive reductions in the intensity of US2 in Experiment 2 of the present study sought to address this possibility. Although a weakening of the forward CR was in fact observed, as predicted by Asratyan's conceptualization, such a decrement was not accompanied by the emergence of the backward CR.

Together, these experiments corroborate and extend the conclusion reached by Gormezano and Tait (1976). The results seriously question the utility of the bidirectional hypothesis as a schema for addressing the backward CR and offer no support for the validity of a phenomenon that has been, so remarkably, a continuing source of interest.

\section{REFERENCES}

ASRatyan, E. A. (1965). Compensatory adaptations, reflex activity, and the brain. Oxford: Pergamon Press.

AsRatyan, E. A. (1967). Some peculiarities in the formation, functioning, and inhibition of conditioned reflexes with two-way connections. Progress in Brain Research, 22, 8-20.

BERTTOFF, J. S. (1965). Neural mechanisms of higher vertebrate be havior (W. T. Liberson, Trans.). Boston: Little, Brown.

CaUtela, J. R. (1965). The problem of backward conditioning. Journal of Psychology, 60, 135-144.

Champion, R. A., Jones, J. E. (1961). Forward, backward and pseudoconditioning of the GSR. Journal of Experimental Psychology, 62, 58-61.

Gormezano, I., \& TAIT, R. W. (1976). The Pavlovian analysis of instrumental conditioning. Pavlovian Joumal of Biological Science, 11, $37-55$.

HALL, J. F. (1976). Classical conditioning and instrumental learning: A contemporary approach. New York: Lippincott.

HaLL, J. F. (1984). Backward conditioning in Pavlovian type studies. Pavlovian Journal of Biological Science, 19, 163-168.

HARLOW, H. F. (1939). Forward conditioning, backward conditioning, and pseudoconditioning in the goldfish. Journal of Genetic Psychology, 55, 49-58.

HeTH, C. D. (1976). Simultaneous and backward fear conditioning as a function of number of CS-UCS pairings. Journal of Experimental Psychology: Animal Behavior Processes, 2, 117-129.

MaCKINTOSH, N. J. (1974). The psychology of animal learning. London: Academic Press.

Mahoney, W. J., \& Ayres, J. J. B. (1976). One-trial simultaneous and backward fear conditioning as reflected in conditioned suppression of licking in rats. Animal Learning \& Behavior, 4, 357-362.

Pavlov, I. P. (1927). Conditioned reflexes: An investigation of the physiological activity of the cerebral cortex (G. V. Anrep, Trans.). London: Oxford University Press.

Razran, G. (1956). Backward conditioning. Psychological Bulletin, $63,55-69$.

Razran, G. (1957). The dominance-contiguity theory of acquisition of classical conditioning. Psychological Bulletin, 54, 1-46.
Sheafor, P. J. (1975). Pseudoconditioned jaw movements of the rabbit reflect associations to contextual background cues. Journal of Ex perimental Psychology: Animal Behavior Processes, 104, 245-260.

SPETCh, M. L., Wilkie, D. M., \& Pinel, J. P. J. (1981). Backward conditioning: A reevaluation of the empirical evidence. Psychological Bulletin, 89, 163-175.

SPOONER, A., \& KeLLOGG, W. N. (1947). The backward conditioning curve. American Journal of Psychology, 60, 321-334

STEPHENS, J. M. (1934). The conditioned reflex as the explanation of habit formation: I. The essential factors in the establishment of the conditioned reflex. Journal of General Psychology, 10, 110-135.

Switzer, S. A. (1930). Backward conditioning of the lid reflex. Journal of Experimental Psychology, 13, 76-97.

Trapold, M. A., Homzie, M., \& Rutledge, E. (1964). Backward conditioning and the UCR latency. Journal of Experimental Psychology, 67, 387-391.

WAGNer, A. R., \& TERRY, W. S. (1975). Backward conditioning to a CS following an expected vs. a surprising UCS. Animal Learning \& Behavior, 3, 370-374.

WENDT, G. R. (1930). Analytical study of the conditioned knee jerk Archives of Psychology, 18, No. 123.

WiCKENS, D. D. (1973). Classical conditioning as it contributes to some basic psychological processes. In F. J. McGuigan \& D. B. Lumsden (Eds.), Contemporary approaches to conditioning and learning. Washington, DC: V. H. Winston.

Wickens, D. D., Meyer, P. M., \& Sullivan, S. N. (1961). Classica CSR conditioning, conditioned discrimination, and interstimulus interval in cats. Journal of Comparative \& Physiological Psychology, 54 $572-576$

Wickens, D. D., Nield, A. F., Tuber, D. S., \& Wickens, C. D (1969). Strength, latency, and form of conditioned skeletal and autonomic responses as functions of CS-UCS intervals. Journal of Experimental Psychology, 80, 165-170.

Wickens, D. D., Tuber, D. S., Nield, A. F., \& Wickens, C. D (1977). Memory for the conditioned response: The effects of potential interference introduced before and after original conditioning. Journal of Experimental Psychology: General, 106, 47-70.

WOLFLE, H. M. (1932). Conditioning as a function of the interval between the conditioned and original stimulus. Journal of General Psychology, 7, 80-103.

\section{NOTE}

1. The characteristics of the forward CR based on this response system have been previously described (D. D. Wickens, Nield, Tuber, \& C. D. Wickens, 1969). Especially critical with regard to the US-US paradigm is the fact that each of the cat's forepaws can be viewed as an independent response system: Not only does the mild shock to one forepaw not activate the contralateral forelimb, but when each forelimb has been conditioned independently to respond with morphologically different characteristics to different stimuli, simultaneous presentation of the stimuli elicits both responses simultaneously (D. D. Wickens, 1973).

(Manuscript received January 6, 1986 revision accepted for publication July 18,1986 .) 